\title{
Alcohol consumption and risk of microvascular complications in type 1 diabetes patients: the EURODIAB Prospective Complications Study
}

\author{
J. W. J. Beulens • J. S. Kruidhof • D. E. Grobbee • \\ N. Chaturvedi - J. H. Fuller • S. S. Soedamah-Muthu
}

Received: 5 March 2008 / Accepted: 17 June 2008 / Published online: 15 July 2008

(C) Springer-Verlag 2008

\begin{abstract}
Aims/hypothesis The aim of this study was to investigate the association between alcohol consumption and risk of microvascular complications (retinopathy, neuropathy, nephropathy) in type 1 diabetes mellitus patients in the EURODIAB Prospective Complications Study.

Methods The EURODIAB Prospective Complications Study is a follow-up study including 3,250 type 1 diabetes mellitus patients from 16 different European countries. We investigated the cross-sectional association between moderate alcohol consumption and risk of retinopathy, neuropathy and nephropathy among 1,857 of these patients.

Results We documented 304 cases of proliferative retinopathy, 660 cases of neuropathy and 157 cases of nephropathy (macroalbuminuria). Alcohol consumption was associated with risk of proliferative retinopathy, neuropathy and macroalbuminuria in a U-shaped fashion. Moderate consumers (30-70 g alcohol per week) had a lower risk
\end{abstract}

Electronic supplementary material The online version of this article (doi:10.1007/s00125-008-1091-z) contains supplementary material, which is available to authorised users.

J. W. J. Beulens $(\bowtie) \cdot J$. S. Kruidhof $\cdot$ D. E. Grobbee

S. S. Soedamah-Muthu

Julius Center for Health Sciences and Primary Care,

University Medical Center Utrecht,

Room STR 6.131, P.O. Box 85500, 3508 GA Utrecht,

The Netherlands

e-mail: J.Beulens@umcutrecht.nl

N. Chaturvedi

National Heart and Lung Institute, Imperial College,

London, UK

J. H. Fuller

Department of Epidemiology and Public Health,

Royal Free and University College London Medical School,

London, UK of microvascular complications with odds ratios of 0.60 (95\% CI 0.37-0.99) for proliferative retinopathy, 0.61 (0.41-0.91) for neuropathy and $0.36(0.18-0.71)$ for macroalbuminuria in multivariate-adjusted models. These results were similar when excluding patients who had been advised to drink less alcohol because of their health. The relation was most pronounced for alcohol consumption from wine. Drinking frequency was significantly, inversely associated with risk of neuropathy, but a similar trend was visible for proliferative retinopathy and macroalbuminuria. Alcohol consumption was not associated with occurrence of ketoacidosis or hypoglycaemic attacks.

Conclusions/interpretation Consistent with its effects on macrovascular complications, moderate alcohol consumption is associated with a lower risk of all microvascular complications among type 1 diabetes patients.

Keywords Alcohol consumption · Macroalbuminuria · Microvascular complications · Nephropathy · Neuropathy · retinopathy Type 1 diabetes

\section{Abbreviation \\ MET metabolic equivalent}

\section{Introduction}

Moderate alcohol consumption, one to three drinks a day (equivalent of 10 to $30 \mathrm{~g}$ alcohol/day), decreases the risk of cardiovascular disease and type 2 diabetes in the general population $[1,2]$. Similarly, diabetes patients who drink in moderation have an approximately $40 \%$ lower risk of cardiovascular disease than abstainers [3]. Consequently, both British and American Diabetes Association guidelines give limits in line with those for the general population in 
terms of alcohol intake and recommend no more than around two units of absolute alcohol at a single sitting [4]. For insulin-treated patients, guidelines emphasise that alcohol should be consumed with food or shortly before eating, as the risk of hypoglycaemia may extend for several hours after drinking [4]. However, apart from macrovascular complications, diabetes patients have an increased risk of developing microvascular complications [5]. It is not fully clear whether alcohol consumption also influences risk of the microvascular complications neuropathy, retinopathy and nephropathy [6].

Only few studies have reported on the relation between alcohol consumption and microvascular complications. Three of these assessed the effect of alcohol consumption on retinopathy with inconsistent results ranging from an inverse relation, no relation and a positive association [79]. Two cross-sectional studies showed no relation between alcohol consumption and the incidence of neuropathy [10, 11], although the incidence of neuropathy was higher in heavy drinkers according to a small cross-sectional study [12]. Finally, another cross-sectional study among 998 patients reported a decreased risk of microalbuminuria for light drinkers [13]. These studies, however, generally had small sample sizes and were limited to type 2 diabetes patients; they also did not quantify the amount of alcohol consumed. The latter issue is of particular importance given the non-linear relationship with macrovascular complications. In addition, the influence of specific alcoholic beverages and drinking frequency on these relations has not been investigated.

To address these issues, we investigated the association between alcohol consumption and risk of microvascular complications, specifically neuropathy, retinopathy and nephropathy, among type 1 diabetes mellitus patients in the EURODIAB Prospective Complications Study.

\section{Methods}

The EURODIAB Prospective Complications Study enrolled 3,250 type 1 diabetes mellitus patients who had been diagnosed before the age of 36 years and had a continuous need for insulin injections within 1 year of diagnosis. Full details on design, methods and recruitment have been published elsewhere [14]. In brief, patients were recruited from 31 centres in 16 European countries between 1989 and 1991. A stratified random sample of up to 140 patients aged 15 to 60 years was selected at each participating clinic from a sampling frame of all type 1 diabetes patients who had attended the clinic at least once during the previous year. Pregnant woman, patients who were not representative of local ethnic groups and those with type 1 diabetes for less than 1 year were excluded from the study. Of the invited patients, $85 \%$ took part in the study. Patients were stratified by sex, age (15-29, 30-44 and 45-60 years) and duration of illness $(1-7,8-14, \geq 15$ years) before ten patients were randomly selected from each stratum. Ethics committee approval was obtained at each centre and all participants provided written informed consent. Participants were invited for re-examination 7 years after baseline examination and both determinant and outcome of this study were measured at this examination. Of the 3,250 patients, 463 were lost to follow-up and 102 died. At follow-up $1,880(70 \%)$ of the remaining 2,635 patients attended the examinations. Data on alcohol consumption were missing for 20 patients and on microvascular complications for three patients, leaving 1,857 patients for analysis. Comparison of baseline characteristics of those included with those excluded did not show any substantial differences between both groups. For retinopathy, neuropathy and nephropathy endpoints were determined for 1,528 , 1,735 and 1,650 patients respectively. If for a certain complication the endpoint was not determined, that patient was excluded from analyses of that complication.

Measurements Blood pressure was recorded in a sitting position with a random zero sphygmomanometer (Hawskley, Lancing, UK) and the mean of two measurements was calculated. If possible, fasting blood samples were taken and sent to a central laboratory for all analyses. $\mathrm{HbA}_{1 \mathrm{c}}$ (normal range $2.9-4.8 \%$ ) was measured by an enzyme immunoassay using a monoclonal antibody raised against $\mathrm{HbA}_{1 \mathrm{c}}$ (Dako, Ely, UK). Plasma levels of triacylglycerol, and total and HDL-cholesterol concentrations were determined by standard enzymatic methods (Boehringer Mannheim, Burgess Hill, UK) using a Cobas-bio centrifugal analyser (Roche, Welwyn Garden City, UK) [15]. For HDL, samples with triacylglycerol concentrations $>3.0 \mathrm{mmol} / \mathrm{l}$ were diluted with $0.15 \mathrm{~mol} / 1$ sodium chloride solution before chemical precipitation. LDL-cholesterol was calculated from Friedewald's formula if triacylglycerol was below $4.5 \mathrm{mmol} / 1$ [15]. Fifteen patients did not have their LDL-cholesterol calculated. Non-HDL-cholesterol was calculated as HDL-cholesterol subtracted from total cholesterol.

Patients filled in a general questionnaire on lifestyle, disease history and family history. Smoking habits and family history of diabetes mellitus were obtained from this questionnaire. Physical activity level was also assessed using this questionnaire, which included questions on (1) how many kilometres patients walked and cycled per day, (2) whether they played a sport and (3) how long and how often they played this sport. Using this information, metabolic equivalent (MET) h per week of physical activity was estimated by multiplying walking, cycling or sporting time per week with their equivalent in MET $\mathrm{h}$ and summing these factors [16]. 
Assessment of alcohol consumption Alcohol intake was assessed using the general questionnaire. Patients were asked if they had ever had an alcoholic drink in their life. If yes, they were asked how often they had usually drunk wine, beer, spirits, sherry or shandy during the last 12 months quantified according to the following categories: (1) almost every day; (2) 3 to 4 days a week; (3) once or twice a week; (4) once or twice a month; (5) once every 2 months; (6) once or twice a year; and (7) never in the past year. Patients were also asked how much wine, beer, spirits, sherry or shandy they usually drank on a drinking day (reported in $\mathrm{ml}$ ). Alcohol intake in $\mathrm{g}$ alcohol per week was determined by multiplying the weekly intake of each alcoholic beverage by its ethanol content (wine 12\%, beer $5 \%$, spirits $35 \%$, sherry $15 \%$ and shandy $0.5 \%$ ) [17] and categorised into six categories: abstainers, 0-5.0 g/week, 5.0-29.9 g/week, 30-69.9 g/week, 70.0-209.9 g/week and $\geq 210 \mathrm{~g} /$ week. The same six categories were used for beer and wine consumption. For spirit consumption, the last two categories were combined because of small sample size. Patients were also asked whether they had been advised to drink less alcohol because of their health. A variable for drinking frequency was created using the responses to the questions on frequency of consuming the separate beverages.

Complications Retinopathy was assessed by taking retinal photographs (two precisely $45^{\circ}$ fields in each eye) after pupil dilatation, which were graded centrally against standard photographs (EURODIAB Hammersmith System) and classified as none, background or proliferative [14]. Nephropathy was diagnosed if AER exceeded $20 \mu \mathrm{g} / \mathrm{min}$ after examination of two $24 \mathrm{~h}$ urine collections. Samples were analysed using an immunoturbidimetric method using goat antihuman albumin antisera and human serum albumin standards (OHRA 20/21 grade HAS; Behring Diagnostics, Hoechst, Milton Keynes, UK) [18]. AER was classified as normoalbuminuria $<20 \mu \mathrm{g} / \mathrm{min}$, microalbuminuria between 20 and $200 \mu \mathrm{g} / \mathrm{min}$ and macroalbuminuria $>200 \mu \mathrm{g} / \mathrm{min}$ [14]. Neuropathy was diagnosed, if two or more of the following symptoms were present: (1) presence of one or more neuropathic symptoms; (2) absence of two or more ankle or knee reflexes; and (3) abnormal vibration perception threshold, measured by centrally calibrated biothesiometers (Biomedical, Newbury, OH, USA) on the right big toe and on the right medial malleolus [11]. We also included the occurrence of ketoacidosis or hypoglycaemic episodes over the past year as endpoints that were reported in the questionnaire.

Statistical analysis Differences between alcohol intake categories were determined by analysis of variance for continuous variables and by a $\chi^{2}$ test for categorical variables. Multinomial logistic regression was used to assess whether alcohol consumption was associated differently with background or proliferative retinopathy and with micro-or macroalbuminuria. Because alcohol consumption was not associated with background retinopathy and microalbuminuria, we only did further investigations on the associations with proliferative retinopathy and macroalbuminuria. Logistic regression was used to determine the association between alcohol consumption and proliferative retinopathy, neuropathy or macroalbuminuria. Three models correcting for different confounding variables were used. The first model was age- and sex-adjusted, while the second model also included centre, smoking (non-, ex-, current), physical activity, duration of diabetes mellitus, systolic blood pressure and BMI. Finally, we added $\mathrm{HbA}_{1 \mathrm{c}}$ to the second model. We conducted tests of linear trend across categories of increasing alcohol consumption by treating the midpoints of consumption in categories as a continuous variable. The square of this term was used to assess the quadratic trend across alcohol consumption categories. We also included total and HDL-cholesterol in the full model to assess whether these markers explain the relation between alcohol consumption and microvascular complications. For beverage-specific analysis we adjusted for all confounders in the third model, but we additionally adjusted for consumption of each of the other beverage types. Associations with drinking frequency were also adjusted for total alcohol consumption. Analyses were repeated excluding patients who reported having changed their alcohol consumption upon doctors' advice and using teetotallers as a separate alcohol intake category. Analyses were performed using the statistical package SPSS 14.0 (SPSS, Chicago, IL, USA). A two-tailed $p$ value of $<0.5$ was considered significant.

\section{Results}

Characteristics of the study population by alcohol consumption are shown in Table 1. Percentage males, BMI, prevalence of smoking, physical activity and systolic blood pressure increased with increasing alcohol consumption, while $\mathrm{HbA}_{1 \mathrm{c}}$ decreased. Alcohol consumption in this population is slightly lower, but within the range of consumption for the general European population [19].

We documented 304 cases of proliferative retinopathy, 660 cases of neuropathy and 157 cases of macroalbuminuria in our study population. The relation between alcohol consumption and proliferative retinopathy, neuropathy and macroalbuminuria is shown in Table 2. We observed a significant $(p=0.019)$ U-shaped association between alcohol consumption and risk of proliferative retinopathy in age- and sex-adjusted models. Similar results $(p=0.021)$ 
Table 1 Characteristics of 1,857 type 1 diabetes patients in the EURODIAB Study by alcohol consumption categories

\begin{tabular}{|c|c|c|c|c|c|c|c|}
\hline \multirow[t]{2}{*}{ Variable } & \multicolumn{6}{|c|}{ Alcohol consumption (g/week) } & \multirow[t]{2}{*}{$p$ value } \\
\hline & 0 & $0-4.9$ & $5.0-29.9$ & $30.0-69.9$ & $70.0-209.9$ & $\geq 210$ & \\
\hline$n$ & 254 & 345 & 390 & 332 & 382 & 154 & \\
\hline Age (years) & $40.6 \pm 10.3$ & $38.8 \pm 10.1$ & $38.5 \pm 9.7$ & $39.9 \pm 9.1$ & $40.6 \pm 9.9$ & $41.4 \pm 9.2$ & 0.036 \\
\hline Sex, male $(\%)$ & 31.1 & 29.6 & 49.2 & 56.6 & 70.9 & 85.7 & $<0.001$ \\
\hline Alcohol consumption (g/week) & & & & & & & $<0.001$ \\
\hline Wine & 0 & $0.8 \pm 1.1$ & $7.4 \pm 7.5$ & $28.2 \pm 20.3$ & $67.2 \pm 55.3$ & $160.2 \pm 170.3$ & \\
\hline Beer & 0 & $0.6 \pm 1.1$ & $6.1 \pm 7.3$ & $16.0 \pm 18.1$ & $44.4 \pm 49.3$ & $198.1 \pm 257.5$ & \\
\hline Spirits & 0 & $0.2 \pm 0.5$ & $1.5 \pm 3.7$ & $3.2 \pm 8.2$ & $9.8 \pm 22.8$ & $46.5 \pm 129.6$ & \\
\hline BMI $\left(\mathrm{kg} / \mathrm{m}^{2}\right)$ & $24.0 \pm 3.5$ & $24.8 \pm 3.7$ & $24.6 \pm 3.3$ & $24.5 \pm 2.9$ & $24.6 \pm 2.8$ & $25.4 \pm 3.3$ & 0.013 \\
\hline $\mathrm{HbA}_{1 \mathrm{c}}(\%)$ & $8.7 \pm 1.6$ & $8.8 \pm 1.7$ & $8.5 \pm 1.5$ & $8.4 \pm 1.3$ & $8.4 \pm 1.5$ & $8.5 \pm 1.4$ & 0.001 \\
\hline WHR & $0.92 \pm 0.19$ & $0.86 \pm 0.15$ & $0.88 \pm 0.14$ & $0.89 \pm 0.16$ & $0.91 \pm 0.13$ & $0.93 \pm 0.11$ & 0.034 \\
\hline Smokers $(\%)$ & & & & & & & $<0.001$ \\
\hline Non & 59.1 & 53.0 & 39.5 & 41.9 & 35.1 & 22.1 & \\
\hline Former & 17.7 & 25.8 & 27.4 & 29.2 & 32.5 & 37.7 & \\
\hline Current & 21.7 & 20.9 & 32.3 & 28.6 & 32.2 & 39.6 & \\
\hline Physical activity (MET/day) & $15.9 \pm 11.5$ & $16.3 \pm 11.7$ & $18.5 \pm 12.3$ & $18.0 \pm 12.4$ & $18.6 \pm 13.3$ & $19.2 \pm 12.9$ & 0.001 \\
\hline Cholesterol (mmol/1) & $5.4 \pm 1.1$ & $5.4 \pm 1.1$ & $5.3 \pm 1.1$ & $5.3 \pm 1.3$ & $5.3 \pm 1.1$ & $5.5 \pm 1.5$ & 0.36 \\
\hline HDL-cholesterol (mmol/l) & $1.7 \pm 0.4$ & $1.6 \pm 0.5$ & $1.6 \pm 0.4$ & $1.7 \pm 0.4$ & $1.7 \pm 0.5$ & $1.6 \pm 0.4$ & 0.52 \\
\hline LDL-cholesterol (mmol/l) & $3.2 \pm 1.1$ & $3.2 \pm 1.0$ & $3.2 \pm 1.1$ & $3.1 \pm 1.0$ & $3.1 \pm 0.9$ & $3.3 \pm 1.1$ & 0.37 \\
\hline Triacylglycerol (mmol/l) & $1.1 \pm 0.7$ & $1.2 \pm 0.8$ & $1.2 \pm 0.6$ & $1.3 \pm 1.4$ & $1.3 \pm 1.0$ & $1.5 \pm 1.1$ & 0.026 \\
\hline Systolic blood pressure (mm Hg) & $119.5 \pm 19.7$ & $119.4 \pm 19.4$ & $119.9 \pm 18.9$ & $122.5 \pm 17.0$ & $121.1 \pm 17.3$ & $127.3 \pm 19.5$ & $<0.001$ \\
\hline CVD (\%) & 10.3 & 9.7 & 7.4 & 7.5 & 6.8 & 7.1 & 0.53 \\
\hline Diabetes duration (years) & $22.7 \pm 9.3$ & $20.9 \pm 9.1$ & $21.3 \pm 8.6$ & $21.9 \pm 8.8$ & $22.6 \pm 9.6$ & $21.4 \pm 8.6$ & 0.66 \\
\hline Family history diabetes (\%) & 24.0 & 21.4 & 19.5 & 20.2 & 21.7 & 17.5 & 0.65 \\
\hline Insulin dose (IU kg ${ }^{-1}$ day $^{-1}$ ) & $0.68 \pm 0.20$ & $0.69 \pm 0.24$ & $0.66 \pm 0.19$ & $0.65 \pm 0.18$ & $0.64 \pm 0.18$ & $0.67 \pm 0.20$ & 0.006 \\
\hline BP-lowering drugs $(\%)$ & 27.2 & 25.8 & 22.3 & 19.9 & 20.4 & 26.6 & 0.10 \\
\hline Lipid-lowering drugs (\%) & 3.5 & 3.8 & 4.1 & 3.0 & 2.6 & 5.2 & 0.29 \\
\hline
\end{tabular}

Unless otherwise stated, values are mean \pm SD.

CVD, cardiovascular disease

were observed in a multivariate-adjusted model with an odds ratio of 0.60 (95\% CI $0.37-0.99)$ for consumers of 30 to $70 \mathrm{~g}$ alcohol per week.

A similar significant U-shaped relation with alcohol consumption was observed for neuropathy in age- and sexadjusted $(p=0.001)$ and multivariate-adjusted models $(p=0.005)$. Moderate alcohol consumers (30-70 g alcohol per week) had an odds ratio of $0.61(0.41-0.91)$. We also observed a similar U-shaped association $(p=0.004)$ for risk of macroalbuminuria, with an odds ratio of $0.36(0.18$ 0.71 ) for participants consuming 30 to $70 \mathrm{~g}$ per week. Further adjustment for total or HDL-cholesterol did not substantially change these results. Similar associations were observed for men and women.

When using teetotallers as a reference category, we observed similar U-shaped associations with alcohol consumption for proliferative retinopathy $(p=0.079)$, neuropathy $(p=0.009)$ and macroalbuminuria $(p=0.008)$. Corresponding odds ratio for consumers of 30 to $70 \mathrm{~g}$ alcohol per week were $0.58(0.33-1.03)$ for proliferative retinopathy, $0.74(0.46-1.18)$ for neuropathy and 0.47 $(0.20-1.09)$ for macroalbuminuria. When excluding patients who had been advised to drink less alcohol $(n=210)$, we observed comparable odds ratios for consumers of 30 to $70 \mathrm{~g}$ alcohol per week, namely $0.62(0.34-1.14)$ for neuropathy ( $p=0.073), 0.64(0.36-1.35)$ for proliferative retinopathy $(p=0.17)$ and $0.35(0.13-0.97)$ for macroalbuminuria $(p=0.021)$.

Associations between specific alcoholic beverages and proliferative retinopathy, neuropathy and macroalbuminuria are shown in Table 3. Beer consumption showed a significant U-shaped relation $(p=0.027)$ with macroalbuminuria only, with an odds ratio of $0.33(0.13-0.81)$ for participants consuming 30 to $70 \mathrm{~g}$ alcohol per week from beer. Beer consumption was not significantly associated with risk of proliferative retinopathy and neuropathy, although a similar trend was observed. Wine consumption was significantly associated with proliferative retinopathy and macroalbuminuria in a U-shaped fashion. Similar results were observed for neuropathy, although this relation did not reach significance $(p=0.089)$. Odds ratios of 0.85 $(0.52-1.37)$ for proliferative retinopathy, $0.57(0.38-0.85)$ for neuropathy and $0.48(0.25-0.93)$ for macroalbuminuria for moderate consumers of 30 to $70 \mathrm{~g}$ alcohol per week 


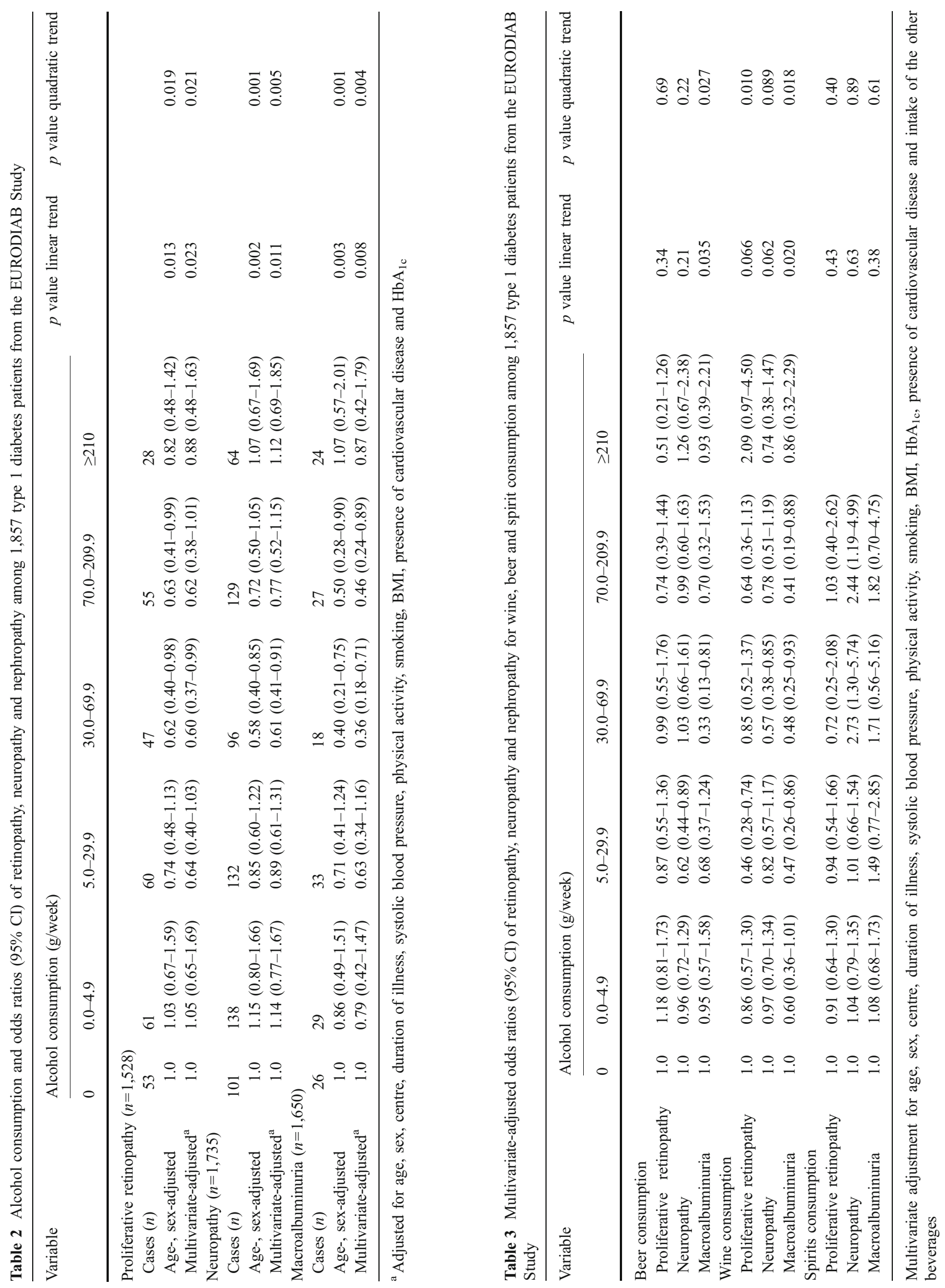


from wine were found. Spirits consumption, however, was not significantly associated with any of the microvascular complications, although it tended to be associated with increased risk. Drinking frequency was inversely associated with risk of neuropathy $(p<0.001)$ and tended to be inversely related to risk of proliferative retinopathy $(p=0.085)$ and macroalbuminuria $(p=0.15)$. Patients consuming alcohol on 5 to 7 days per week had odds ratios of $0.49(0.34-0.71)$ for neuropathy, $0.75(0.48-1.18)$ for proliferative retinopathy and $0.64(0.35-1.14)$ for macroalbuminuria. However, it was difficult to assess the relative importance of drinking frequency and quantity consumed per drinking day, because too little variation in drinks per drinking day existed to draw valid conclusions. Only for neuropathy did we observe a slightly increased risk (OR 1.56 ; 95\% CI $0.66-3.71$ ) for patients consuming $>140 \mathrm{~g}$ alcohol per week on 1 to 2 days, while those consuming 0 to $140 \mathrm{~g}$ alcohol per week on $>3$ days had the lowest risk (OR 0.61; 95\% CI 0.43-0.85). For proliferative retinopathy and macroalbuminuria, we were unable to distinguish such patterns.

Finally, alcohol consumption was not associated with occurrence of ketoacidosis $(p=0.28)$ or hypoglycaemic attacks $(p=0.34)$ over the past year.

\section{Discussion}

This study showed a U-shaped relationship between alcohol consumption and risk of proliferative retinopathy, neuropathy and macroalbuminuria. Moderate alcohol consumers had a decreased risk of microvascular complications compared with abstainers and heavier drinkers. These relations were most pronounced for consumption of wine, while drinking frequency was only inversely associated with neuropathy.

Our study is the first to comprehensively investigate the relation between alcohol consumption and all microvascular complications, while accounting for alcohol dose, beverage type and drinking frequency. Strengths include its large sample size and the elaborate assessment of alcohol consumption, but certain limitations need to be addressed. First, our study had a cross-sectional design, making it difficult to distinguish cause and effect, since patients may have changed their drinking habits and other lifestyle factors after the diagnosis of type 1 diabetes. We re-analysed our data excluding all patients who had been advised to drink less because of their health and using teetotallers as a reference category with similar results. It is therefore unlikely that these effects influenced our results to a large extent. Second, only patients with valid data at follow-up were included ( $\sim 60 \%$ of the entire cohort), but we could not find substantial differences in characteristics between those included and those excluded. This selection, therefore, is unlikely to affect our results to a large extent. Third, we relied on self-reported assessment of alcohol consumption. Feunekes et al. [20] have reviewed the validity of self-reported assessment of alcohol consumption and concluded that such methods are valid to rank persons on the basis of their alcohol consumption. In addition, methods enquiring about the frequency and amount consumed, for beer, wine and liquor, separately, as used in our study, will yield the most valid results. We therefore believe that the method used in our study is valid for the purpose of this study. Finally, our study population consisted of type 1 diabetes mellitus patients only, while the majority of diabetes patients have type 2 diabetes. This limits the generalisability of our results to type 2 diabetes patients.

The results of our study are consistent with the decreased risk of macrovascular complications with moderate alcohol consumption [3] and suggest that moderate alcohol consumption is associated with a generalised cardiovascular protective effect among diabetes patients. On the other hand, certain studies showed slightly different results for particular microvascular complications. For retinopathy, our results are broadly similar to those of Giuffre et al. [7] and Moss et al. [8]. The first observed a decreased risk of retinopathy with increasing duration of alcohol consumption [7], while the latter also observed a slightly decreased risk of retinopathy with light drinking, but this relation was not statistically significant [8]. Finally, Young et al. showed a higher grading of retinopathy for those consuming more than ten alcoholic drinks per week compared with those drinking less than ten drinks per week [9]. For neuropathy, Franklin et al. observed an odds ratio of 0.71 for moderate consumers of 0 to $20 \mathrm{~g}$ per week, which was similar to our results [10]. Tesfaye et al. [11], on the other hand, did not find an association, but detailed results of this analysis were not reported. In line with our findings, Klein et al. reported an odds ratio of 0.76 for microalbuminuria for light alcohol consumption, but they did not report the exact quantity defined as light drinking [13].

Interestingly, alcohol consumption was not associated with microalbuminuria or background retinopathy. This could be due to the fact that these are less manifest presentations of the complications, making it more difficult to accurately assess these measures. Possibly, effects of alcohol consumption may only be apparent for more manifest outcomes of the disease. Other studies on diabetic retinopathy did not report different effects of alcohol consumption on background or proliferative retinopathy [7-9]. For microalbuminuria, however, Klein et al. [13] did report a relation among type 2 diabetes patients.

The reduced risk of microvascular complications with moderate alcohol consumption was most pronounced for 
wine consumption. Beer consumption was only significantly associated with macroalbuminuria, but similar trends were observed for neuropathy and proliferative retinopathy. Spirits consumption was not associated with any of the microvascular complications. These results suggest an additional favourable effect of drinking moderate amounts of wine, apart from the effect of alcohol itself. Alcohol itself has favourable effects on HDL-cholesterol, inflammation and inhibition of platelet aggregation [21, 22]. The additional favourable influences of wine may be due to phenols, which may decrease platelet aggregation and inhibit HDL-cholesterol oxidation [23]. On the other hand, previous studies have shown that wine drinkers appear to differ from drinkers of other beverages in a variety of characteristics such as diet [24]. A healthier diet could therefore account for the more pronounced results for wine consumption. Finally, wine was the predominant beverage type in our study and these analyses had more power to detect an association. Our findings therefore seem consistent with the hypothesis that the beverage most widely consumed by a certain population is most likely to be inversely associated with the risk of cardiovascular disease in that sample [25]. Spirits consumption, however, was more likely to increase risk of microvascular complications in our study.

The associations with drinking frequency are in line with results found for drinking patterns in the general population for cardiovascular disease [26], but we had too little variation to distinguish between quantity and frequency. Despite this, our results for retinopathy suggest that a drinking pattern characterised by consuming alcohol on several days of the week in moderation is also a healthy pattern for microvascular complications as opposed to episodic drinking.

The similarities of these associations with those for macrovascular complications or with cardiovascular disease in the general population would suggest a similar underlying mechanism. However, adjusting for HDL-cholesterol did not substantially change our estimates, while this is thought to be the most important intermediate for the relation between alcohol consumption and cardiovascular disease [21]. We also adjusted for $\mathrm{HbA}_{1 c}$ concentrations, but this did not change our results. Therefore other mechanisms such as effects on inflammation or platelet aggregation could be involved [21, 22]. However, more research is needed to address these questions.

Consistent with findings for macrovascular complications, our study shows that moderate alcohol consumption also decreases the risk of all microvascular complications among type 1 diabetes patients. This suggests that moderate alcohol consumption is associated with a generalised cardiovascular protective effect among diabetic patients.
Acknowledgements The EURODIAB Prospective Complications Study was supported by grants from the Wellcome Trust, the European Community and Diabetes UK. For details of the EURODIAB centres and investigators, see Electronic supplementary material (ESM).

Duality of interest The authors declare that there is no duality of interest associated with this manuscript.

\section{References}

1. Koppes LL, Dekker JM, Hendriks HF, Bouter LM, Heine RJ (2005) Moderate alcohol consumption lowers the risk of type 2 diabetes: a meta-analysis of prospective observational studies. Diabetes Care 28:719-725

2. Maclure M (1993) Demonstration of deductive meta-analysis: ethanol intake and risk of myocardial infarction. Epidemiol Rev 15:328-351

3. Koppes LL, Dekker JM, Hendriks HF, Bouter LM, Heine RJ (2006) Meta-analysis of the relationship between alcohol consumption and coronary heart disease and mortality in type 2 diabetic patients. Diabetologia 49:648-652

4. American Diabetes Association (2000) Nutrition recommendations and principles for people with diabetes mellitus. Diabetes Care 23(Suppl 1):S43-S46

5. Chaturvedi N (2007) The burden of diabetes and its complications: trends and implications for intervention. Diabetes Res Clin Pract 76(Suppl 1):S3-S12

6. Howard AA, Arnsten JH, Gourevitch MN (2004) Effect of alcohol consumption on diabetes mellitus: a systematic review. Ann Intern Med 140:211-219

7. Giuffre G, Lodato G, Dardanoni G (2004) Prevalence and risk factors of diabetic retinopathy in adult and elderly subjects: The Casteldaccia Eye Study. Graefes Arch Clin Exp Ophthalmol 242:535-540

8. Moss SE, Klein R, Klein BE (1994) The association of alcohol consumption with the incidence and progression of diabetic retinopathy. Ophthalmology 101:1962-1968

9. Young RJ, McCulloch DK, Prescott RJ, Clarke BF (1984) Alcohol: another risk factor for diabetic retinopathy? Br Med J (Clin Res Ed) 288:1035-1037

10. Franklin GM, Shetterly SM, Cohen JA, Baxter J, Hamman RF (1994) Risk factors for distal symmetric neuropathy in NIDDM. The San Luis Valley Diabetes Study. Diabetes Care 17:1172-1177

11. Tesfaye S, Stevens LK, Stephenson JM et al (1996) Prevalence of diabetic peripheral neuropathy and its relation to glycaemic control and potential risk factors: the EURODIAB IDDM Complications Study. Diabetologia 39:1377-1384

12. McCulloch DK, Campbell IW, Prescott RJ, Clarke BF (1980) Effect of alcohol intake on symptomatic peripheral neuropathy in diabetic men. Diabetes Care 3:245-247

13. Klein R, Klein BE, Moss SE (1993) Prevalence of microalbuminuria in older-onset diabetes. Diabetes Care 16:1325-1330

14. No authors listed (1994) Microvascular and acute complications in IDDM patients: the EURODIAB IDDM Complications Study. Diabetologia 37:278-285

15. Soedamah-Muthu SS, Chaturvedi N, Toeller M et al (2004) Risk factors for coronary heart disease in type 1 diabetic patients in Europe: the EURODIAB Prospective Complications Study. Diabetes Care 27:530-537

16. Ainsworth BE, Haskell WL, Whitt MC et al (2000) Compendium of physical activities: an update of activity codes and MET intensities. Med Sci Sports Exerc 32:S498-S504 
17. de Vries JHM, Lemmens PHHM, Pietinen P, Kok FJ (1999) Assessment of alcohol consumption. In: Macdonald I (ed) Health issues related to alcohol consumption. 2nd edn. Blackwell Science, Oxford, pp 1-25

18. Kearney EM, Mount JN, Watts GF, Slavin BM, Kind PR (1987) Simple immunoturbidimetric method for determining urinary albumin at low concentrations using Cobas-Bio centrifugal analyser. J Clin Pathol 40:465-468

19. Sieri S, Agudo A, Kesse E et al (2002) Patterns of alcohol consumption in 10 European countries participating in the European Prospective Investigation into Cancer and Nutrition (EPIC) project. Public Health Nutr 5:1287-1296

20. Feunekes GI, van ‘t Veer P, Van Staveren WA, Kok FJ (1999) Alcohol intake assessment: the sober facts. Am J Epidemiol 150:105-112

21. Rimm EB, Williams P, Fosher K, Criqui M, Stampfer MJ (1999) Moderate alcohol intake and lower risk of coronary heart disease: meta-analysis of effects on lipids and haemostatic factors. BMJ 319:1523-1528
22. Sierksma A, van der Gaag MS, Kluft C, Hendriks HF (2002) Moderate alcohol consumption reduces plasma C-reactive protein and fibrinogen levels; a randomized, diet-controlled intervention study. Eur J Clin Nutr 56:1130-1136

23. Gronbaek M (2006) Factors influencing the relation between alcohol and cardiovascular disease. Curr Opin Lipidol 17:1721

24. Barefoot JC, Gronbaek M, Feaganes JR, McPherson RS, Williams RB, Siegler IC (2002) Alcoholic beverage preference, diet, and health habits in the UNC Alumni Heart Study. Am J Clin Nutr 76:466-472

25. Rimm EB, Klatsky A, Grobbee D, Stampfer MJ (1996) Review of moderate alcohol consumption and reduced risk of coronary heart disease: is the effect due to beer, wine, or spirits. BMJ 312:731736

26. Mukamal KJ, Conigrave KM, Mittleman MA et al (2003) Roles of drinking pattern and type of alcohol consumed in coronary heart disease in men. N Engl J Med 348:109-118 